\title{
Análisis de la productividad científica de la revista Enfermería Universitaria de la Escuela Nacional de Enfermería y Obstetricia (ENEO) de 2007-2011: número y tipo de artículos publicados
}

\author{
María Guadalupe Ortega Ríos, * Lasty Balseiro Almario, ** \\ Federico Sacristán Ruíz,*** Gandhy Ponce Gómez, **** Silvia Hernández Rosas****
}

\begin{abstract}
RESUMEN
El Objetivo de esta investigación fue analizar la productividad científica de la Revista Enfermería Universitaria de la Escuela Nacional de Enfermería y Obstetricia durante el 2007 a 2011, con base en indicadores bibliométricos. Marco teórico: La publicación científica en el ámbito de las ciencias de la salud constituye un indicador importante, una herramienta básica en el desarrollo de las profesiones, dado que la ciencia se da a conocer a partir de la publicación científica, ya que constituye el medio para difundir los conocimientos, contrastar los hallazgos encontrados y utilizarlos en la construcción teórica o práctica de la ciencia. Para medir la productividad científica, se utiliza la bibliometría que permite con base en los indicadores bibliométricos medir la calidad editorial y científica de una revista. Metodología: La variable medida fue la calidad editorial y científica. En esta investigación, se midió la dimensión de la productividad científica con base en los indicadores bibliométricos. El tipo de estudio fue de corte cuantitativo no experimental, retrospectivo, transversal, comparativo, analítico, descriptivo y diagnóstico. Resultados: Del 2007 a 2011 se produjeron 20 revistas, de las cuales tres se editaron en el 2007, cuatro números en cada uno en los años 2008, 2009, y 2011, de los cuales se publicaron un total de 148 artículos. Conclusiones: La Revista Enfermería Universitaria ha mejorado considerablemente su calidad editorial, lo que le ha permitido mantener su posición en el ámbito nacional e internacional, y ser reconocida como un órgano importante para la difusión y la comunicación científica del quehacer de la enfermería.
\end{abstract}

Palabras clave: Evaluación, revista, enfermería universitaria, productividad científica, indicadores bibliométricos, publicaciones.

\section{Scientific productivity analysis of college nursing journal of National School of Nursing and Obstetrics (ENEO) 2007-2011: number and type of articles}

\footnotetext{
ABSTRACT

The Objective of this research was to analyze the scientific productivity of the College Nursing Journal of the National School of Nursing and Obstetrics (ENEO) during 2007-2011, based on bibliometric indicators. Theoretical framework:

* Pasante de la Licenciatura de Enfermería y Obstetricia de la UNAM.

** Responsable del Proyecto de Investigación Folio 067 del Comité de Investigación de la ENEO.

*** Corresponsable del Proyecto de Investigación Folio 067 del Comité de Investigación de la ENEO. ***** Colaboradores del Proyecto.

Correspondencia: Dra. Lasty Balseiro Almario. Antiguo Camino Viejo a Xochimilco s/n Viaducto Tlalpan, Col. San Lorenzo Huipulco, C.P. 14370, México, D.F. E- mail: lastybals@hotmail.com 
The scientific publication in the field of health sciences is an important indicator, an essential tool in the development of the professions, since science is disclosed from scientific publication and bibliometric indicators, because it is the means to disseminate knowledge, contrasting the findings and use them in the theoretical or practical construction of science. To measure scientific productivity is used the bibliometry, which allows based on bibliometric indicators measure the quality and scientific editorial of a journal. Methodology: The measured variable was scientific and editorial quality. In this research we measured the size of scientific productivity, based on bibliometric indicators. The type of study was a quantitative non-experimental, retrospective, cross-sectional, comparative, analytical, descriptive and diagnostic. Results: From 2007 to 2011 there were 20 journals, of which three were edited in 2007, four issues in each of the years 2008, 2009 and 2011 and published a total of 148 articles. Conclusions: College Nursing Journal has considerably improved its editorial quality which has enabled it to maintain its position in the national and international recognition as an important organ for the diffusion of scientific communication and practice of nursing.

Key words: Evaluation, journal, nursing college, scientific productivity, bibliometric indicators, publications.

\section{INTRODUCCIÓN}

El objetivo de esta investigación fue analizar la productividad científica de la revista Enfermería Universitaria de la ENEO durante los años 2007-2011 con base en indicadores bibliométricos. Los objetivos específicos fueron examinar el número y tipo de artículos publicados por año.

\section{MARCO TEÓRICO}

Para Manuel Amezcua, ${ }^{1}$ la construcción de la ciencia implica un proceso complejo, cuyo resultado final es una publicación. Así, la ciencia se da a conocer a partir de la publicación científica, ya que éste es el medio para difundir los conocimientos, contrastar los hallazgos encontrados y utilizar estos hallazgos en la construcción teórica o práctica de la ciencia. La ciencia, entonces, es un producto que se publica y se remite al estudio de una actividad llamada científica que refleja el pensamiento de esta comunidad, que pone al servicio de todos los hallazgos encontrados. Por ello, en una comunidad internacional que está globalizada, el conocimiento científico está al alcance de todos.

Por su parte, para Lourdes García Suárez, ${ }^{2}$ la publicación científica en el ámbito de las ciencias de la salud constituye un indicador importante y una herramienta básica en el desarrollo de sus profesionales y en la elevación de la salud del pueblo. De ahí que se deba garantizar su validez y oportunidad, así como el cumplimiento de otros atributos que dan cuenta de su calidad. Tal es el caso del cumplimiento de las normas que dicta la ética de las publicaciones científicas. Para Gualberto Buela-Casal, ${ }^{3}$ la evaluación de las publicaciones científicas debería realizarse a dos niveles: artículos y revistas, siguiendo los criterios mencionados anteriormente. En un primer nivel, tal como se suele realizar en la actualidad con la participación de comités, aunque sería conveniente que éstos fuesen mejor seleccionados y quizá debería plantearse también la cuestión de que las evaluaciones sean anónimas. En un segundo nivel, se pueden evaluar las revistas por un comité mixto de expertos profesionales. Cada revista se evaluaría anualmente y recibiría una puntuación sobre la calidad.

Por otra parte, para medir la productividad se utiliza la Bibliometría que es la ciencia que permite el análisis cuantitativo de la producción científica a través de la literatura, estudiando la naturaleza y el curso de una disciplina científica. Los indicadores bibliométricos se utilizan ampliamente para la evaluación de investigadores, instituciones y países, pero los datos que nos arrojan no deben interpretarse de manera aislada como se hace frecuente con el factor impacto. ${ }^{4}$ De igual forma, para María Isabel Orts, ${ }^{5}$ la producción científica, en términos bibliométricos, es la suma de productos científicos individualizados generados por una determinada comunidad (nacional, internacional, sectorial) durante un periodo convenido, y físicamente identificables como publicaciones, cualquiera que sea su forma: artículos y notas aparecidas en revistas, monografías, libros, ponencias presentadas a congresos, etc.

Una herramienta utilizada para evaluar dicha producción científica, y más concretamente el estudio de las publicaciones científicas, es la Bibliometría. Así, los indicadores bibliométricos son "datos numéricos sobre fenómenos sociales de la actividad científica relativos a la producción, transmisión y consumo de la información en el seno de comunidades determinadas". Los indicadores bibliométricos se clasifican en cuatro categorías correspondientes a la producción (número de artículos, autores, revistas), a la circulación y dispersión (análisis de las bases de datos de información bibliográfica y de la distribución de la literatura a distintas disciplinas), al consumo (análisis de las referencias) y a la repercusión (análisis de las citas) de las publicaciones y de la información que contienen. Así, para L. Ferreiro (1993), ${ }^{6}$ la "Bibliometría es el método 
científico de la ciencia de la información o ciencia de la documentación y constituye la aproximación cuantitativa que permite el desarrollo de la teoría general de esta ciencia y el estudio descriptivo e inferencial o inductivo de todas las formas de la comunicación escrita, adoptadas por la literatura científica". La Bibliometría se encarga, por tanto, del estudio de los aspectos cuantitativos de la producción científica, así como de su diseminación y uso. Para llevar a cabo esta función, emplea modelos y medidas matemáticas que permiten cuantificar la información bibliográfica susceptible de ser analizada (Garfield, 1978). Para Alberto Gálvez Toro, ${ }^{7}$ la globalización del conocimiento en el campo de la enfermería ha demostrado que hay comunidades científicas cuya producción es poco visible más allá de sus fronteras, mientras que otras comunidades ejercen un poder hegemónico en el ámbito de la comunicación científica. Esto es lo que ocurre a las comunidades lingüísticas del español y del portugués frente a las comunidades que utilizan el inglés como medio de transmisión del conocimiento. Esta es una observación común entre los investigadores que se expresan en español y en portugués. Así, las enfermeras españolas desconocen la existencia de revistas brasileñas o mexicanas, las enfermeras colombianas consumen poca prensa científica española, argentina o mexicana. Sin embargo, todas estas enfermeras consumen abundante literatura periódica anglosajona. Es absurdo e incluso contradictorio que comunidades científicas cultural y lingüísticamente próximas y con una producción científica de calidad, carezcan de un conocimiento mutuo y de una transferencia efectiva del conocimiento entre ellas. Obviamente, esta conducta es negligente y supone un menosprecio del valor del conocimiento propio.

Por ello, al realizar la evaluación interna de la revista, midiendo la productividad científica con base en los indicadores bibliométricos, daremos cuenta de los aspectos cuantitativos de esta producción y de cómo las enfermeras mexicanas están realizando literatura científica para mostrar la calidad de su productividad.

\section{METODOLOGÍA}

La variable medida fue calidad editorial y científica de la revista Enfermería Universitaria. Se realizó una evaluación interna. Las dimensiones de la variable para la evaluación interna de la revista fueron: productividad, citación, visibilidad, obsolescencia y áreas del conocimiento de enfermería. En cada una de las dimensiones de la variable se analizaron sus indicadores, en el caso de la dimensión de productividad científica que se describe en este artículo se consideraron los siguientes: el número de revistas y de artículos publicados, por año y por revista. Según el tipo de artículo publicado: editorial, de opinión, de revisión, ensayo o reseña. En cuanto al personal académico de la ENEO que publica en la revista, se identificó la división de adscripción: Estudios de Posgrado, Estudios Profesionales, Educación Continua y el Sistema de Educación Abierta y a Distancia (SUAyED).

El diseño metodológico fue de corte cuantitativo no experimental, retrospectivo, transversal, comparativo, analítico, descriptivo y diagnóstico. La muestra de estudio fue el total de artículos de revistas editadas desde los años 2007 hasta 2011. Como criterio de inclusión, se consideraron todos los números de la revista Enfermería Universitaria publicados en esos años. Se excluyeron todos los artículos de las revistas publicadas en los números anteriores a 2007 y posteriores a 2011. En esta investigación, ningún artículo, sección o número de revista publicado en el periodo citado fue eliminado. Las técnicas consideradas en la evaluación interna fueron la observación y el análisis directo mediante una revisión detallada de cada una de las revistas.

En el proceso de evaluación interna se hizo un análisis exhaustivo de cada una de las revistas, ordenando y clasificando los datos en una base, para la aplicación posterior del paquete estadístico SPSS v.14 y análisis de la información. En los lineamientos éticos no se consideró ningún riesgo y, desde luego, se tomó en cuenta el principio de anonimato y privacidad de autores, suscriptores y jueces, conforme al reglamento de la Ley General de Salud en materia de investigación (1987) título II, capítulo XIII, XVI y C de la Secretaría de Salud que se refiere al principio de la privacidad y en donde prevalece el criterio de respeto a la dignidad y a la protección de los derechos y bienestar de los seres humanos.

Los recursos utilizados en esta investigación corresponden a los humanos con dos profesores de carrera de tiempo completo: uno titular B, y otro asociado C; dos pasantes egresados de la licenciatura en Enfermería y Obstetricia y uno de la licenciatura en Enfermería. Los recursos físicos y tecnológicos fueron los propios de la División de Estudios de Posgrado de la ENEO, específicamente de la Unidad de Investigación. Por último, el aspecto financiero fue cubierto por el grupo de investigación.

\section{RESULTADOS}

Respecto a la dimensión productividad científica, se obtuvo que de 2007 a 2011 se produjeron 20 revistas, de las cuales tres se editaron en el 2007, cuatro números en cada uno de los años 2008, 2009, y 2011, excepto en el año 2010 cuando se editaron cinco números, uno de ellos extraordinario dedicado al aniversario de la Revolución Mexicana (Cuadro I). Se publicaron 148 artículos, todos 
en idioma español, distribuidos por año y número de revista: 30 fueron en el 2007, 31 en el 2008, 27 en 2009, 33 en 2010 y 27 en el 2011 (Cuadro II). Respecto al tipo de artículos publicados, el $13.64 \%$ (20) fueron editoriales, el $11.47 \%$ (11) de opinión, el 5.40\% (8) de revisión; el 4.72\% (7) ensayos; el $1.35 \%$ (2) de reseña. Según el campo de conocimiento, el $41.62 \%$ (61) fueron artículos de investigación; el 19.59\% (29) de innovación a la práctica, y con un $6.70 \%$ (10), los de docencia. No se publicaron artículos de estudios de caso (Cuadro III).

\section{DISCUSIÓN}

Las revistas científicas desempeñan un papel importante en el proceso de comunicación científica, ya que constituyen la principal vía para la validación del nuevo conocimiento y hacen posible su difusión dentro de la comunidad de

Cuadro I. Número de revistas de Enfermería Universitaria producidas por año, de 2007 a 2011 (México, D.F., 2013).

\begin{tabular}{ccc}
\hline Año de la Revista & Núm. de revistas por año & $\%$ \\
\hline 2007 & 3 & 15 \\
2008 & 4 & 20 \\
2009 & 4 & 20 \\
2010 & 5 & 25 \\
2011 & 4 & 20 \\
Total & 20 & $100 \%$ \\
\hline
\end{tabular}

Fuente: Revista Enfermería Universitaria. investigadores, siendo el principal medio de comunicación en muchas áreas científicas. La comunicación científica se realiza tradicionalmente a través de la publicación de artículos en revistas especializadas, que constituyen el vehículo por excelencia de la comunicación del conocimiento científico. Por este motivo, el estudio de las publicaciones de un centro, área, comunidad o país es un indicador válido para cuantificar la actividad científica de dichas unidades. ${ }^{8}$ En este contexto, la revista Enfermería Universitaria de la ENEO se ha constituido en una de las fuentes de información de la disciplina de enfermería que dan cuenta de la práctica profesional, por lo que es considerada como un referente de consulta para los profesionales en ejercicio y los recursos humanos en formación. Así lo avalan sus 20 revistas con 148 artículos en las publicaciones que han sido periódicas y continuas durante los cinco años del lapso evaluado, lo que coincide con el estudio de Serrano ${ }^{9}$ de la revista Metas de Enfermería, en el cual señala que dar a conocer los hallazgos de las investigaciones es de crucial importancia para que se siga produciendo conocimiento científico. Sin duda, las publicaciones periódicas son el vehículo por excelencia para este menester. Las publicaciones periódicas constituyen actualmente la fuente de información de cualquier disciplina, dado que en ellas se vierten los contenidos de mayor vigencia, y permiten estar al día en todo aquello que se necesita para una práctica profesional de calidad. Al respecto, María Bordons y María Ángeles ${ }^{10}$ plantean que el número de publicaciones de un

Cuadro II. Número de artículos publicados por año en la revista Enfermería Universitaria, de 2007 a 2011 (México, D.F., 2013 ).

\begin{tabular}{|c|c|c|c|c|c|c|c|}
\hline Año & Núm. 1 & Núm. 2 & Núm. 3 & Núm. 4 & Núm. 5 & Total & $\%$ \\
\hline 2007 & 11 & 12 & 7 & 0 & 0 & 30 & 20.27 \\
\hline 2008 & 8 & 8 & 8 & 7 & 0 & 31 & 20.94 \\
\hline 2009 & 7 & 6 & 7 & 7 & 0 & 27 & 18.25 \\
\hline 2010 & 5 & 6 & 7 & 8 & 7 & 33 & 22.29 \\
\hline 2011 & 8 & 7 & 7 & 5 & 0 & 27 & 18.25 \\
\hline Total & 39 & 39 & 36 & 27 & 7 & 148 & 100.00 \\
\hline
\end{tabular}

Fuente: Revista Enfermería Universitaria.

Cuadro III. Tipo de artículo publicado en la revista Enfermería Universitaria de 2007 a 2011 (México, D.F., 2013).

\begin{tabular}{|c|c|c|c|c|c|c|c|c|c|c|c|}
\hline Año & Editorial & Investigación & Opinión & Revisión & $\begin{array}{l}\text { Estudio } \\
\text { de caso }\end{array}$ & Ensayo & $\begin{array}{l}\text { Innovación a } \\
\text { la práctica }\end{array}$ & Reseña & Docencia & Fo & $\%$ \\
\hline 2007 & 3 & 8 & 6 & 0 & 0 & 7 & 6 & 0 & 0 & 30 & 20.28 \\
\hline 2008 & 4 & 13 & 3 & 0 & 0 & 0 & 7 & 0 & 4 & 31 & 20.95 \\
\hline 2009 & 4 & 12 & 0 & 0 & 0 & 0 & 5 & 0 & 6 & 27 & 18.24 \\
\hline 2010 & 5 & 17 & 2 & 2 & 0 & 0 & 5 & 2 & 0 & 33 & 22.29 \\
\hline 2011 & 4 & 11 & 0 & 6 & 0 & 0 & 6 & 0 & 0 & 27 & 18.24 \\
\hline Total & 20 & 61 & 11 & 8 & 0 & 7 & 29 & 2 & 10 & 148 & $100 \%$ \\
\hline$\%$ & 13.64 & 41.62 & 7.43 & 5.40 & 0 & 4.72 & 19.59 & 1.35 & 6.70 & $100 \%$ & \\
\hline
\end{tabular}

Fuente: Revista Enfermería Universitaria. 
centro, área o país es un indicador útil para cuantificar la actividad científica de dichas unidades. Su mayor utilidad se obtiene al efectuar comparaciones con la actividad de otros centros, áreas o países pues se hace necesario tener un marco de referencia en el que se pueda ubicar nuestro objeto de estudio; también es interesante realizar seguimientos de la producción científica a lo largo del tiempo.

En cuanto al tipo de artículos publicados en la revista Enfermería Universitaria, el $41.62 \%$ corresponden a investigación; $19.59 \%$ son de innovación a la práctica; $13.64 \%$ a editoriales; $11.47 \%$ de opinión; $4.72 \%$ a ensayos; $1.35 \%$ a reseñas y $6.70 \%$ de docencia. En estas cifras destaca que no se publicaron estudios de caso, lo que significa un elemento restrictivo en la aportación de enfermería y en su contribución a la publicación de esta metodología. Estos datos contrastan con los del análisis cienciométrico de la revista Enfermería Neurológica de Martín Rodríguez y Cristina Toledo, ${ }^{11}$ quienes encontraron que los artículos más publicados en esta revista corresponden a los de revisión (34\%) seguido de artículos de investigación (64\%), programa y protocolos (14.55\%), de opinión (9.09\%), y de técnicas y procedimiento $(3.64 \%)$.

En otro estudio bibliométrico realizado por Diego Camps ${ }^{12}$ en la Revista Universitaria Médica durante el periodo 2002-2006, se detectó que los porcentajes de artículos publicados comparados con el resto de artículos es bajo si se compara con los de otras revistas, en los que se estima que $50 \%$ son artículos originales. Sin embargo, es difícil la comparación en este ítem por varios factores. Al analizar la contribución científica por autores a la revista Enfermería Universitaria, el $77.80 \%$ han escrito un artículo, el $16.70 \%$, dos artículos; el $2.71 \%$, tres artículos, y el $1.85 \%$, que son sólo dos autores, han escrito cinco artículos. Esto da idea que la gran mayoría de los autores publican una vez en la revista dado que también existen otras revistas donde también pueden publicar. Estas cifras son similares al estudio realizado de la producción científica de la revista Enfermería Neurológica y publicado por Martín Rodríguez y Cristina Toledo, ${ }^{13}$ en el que plantean que al analizar la autoría y colaboración, no encontraron autores únicos por artículo, en ningún número de los publicados se llegó a una media de tres autores (la media más elevada está en el núm. 0 con un promedio de 2.7 de autores firmando el artículo). La endogamia editorial bruta (artículos publicados por miembros del comité asesor de la revista), la sitúa en un $42.27 \%$, aunque es necesario tener en cuenta que en su análisis consideran secciones como casos clínicos, historia de la enfermería y metodología enfermera con producción fija de colaboradores. Sin embargo, para Eduardo Figueredo ${ }^{14}$ en los artículos previos en los que se evalúa la producción científica, se manifiesta que el simple recuento de publicaciones de cada investigador es un indicador poco fiable para medir las aportaciones que ha hecho al progreso de la ciencia. Por tal motivo, sería deseable encontrar indicadores que puedan medir de forma más pormenorizada la contribución individual de cada investigador.

\section{CONCLUSIONES}

La evaluación de la actividad científica medida con indicadores bibliométricos se ha constituido en los últimos años como los instrumentos útiles para estudiar los resultados de la producción científica a través de las publicaciones en las revistas. Por ello, la evaluación de la producción científica de la revista Enfermería Universitaria tiene la intención de identificar aciertos e insuficiencias a fin de apoyar la toma de decisiones para su mejora y fincar nuevas trayectorias para la investigación. Es destacable señalar que el $41.62 \%$ de los artículos publicados correspondieron al campo de investigación, lo cual está acorde con los propósitos y la clasificación de la revista de la ENEO, catalogada como científica, ya que promueve la divulgación del conocimiento de la disciplina en ese ámbito. No obstante, es deseable también que se incremente el número de publicaciones en este rubro.

Por otra parte, respecto a los estudios de caso, se debe exhortar e invitar a los especialistas de enfermería para que publiquen sus experiencias, ya que éstas constituyen una metodología en el ejercicio práctico del cuidado y que, por tanto, aportan evidencias para enriquecer y consolidar el conocimiento que sustenta el quehacer de la enfermería. En los años evaluados de la revista Enfermería Universitaria se ha podido constatar que si se continúa mostrando la literatura científica de las enfermeras(os), al difundir sus conocimientos, tanto a nivel nacional como internacional, será considerada aun más como un referente de consulta para los profesionales en ejercicio y los recursos humanos en formación.

\section{BIBLIOGRAFÍA}

1. Balseiro L y cols. Evaluación interna y externa de la revista Enfermería Universitaria de la ENEO-UNAM durante el periodo 2006-2006. Revista Enfermería Universitaria. 2009; 6 (1): 13.

2. García SL y cols. Análisis bibliométrico de los artículos científicos publicados en la Revista Médica Electrónica (2003-2009). Revista Médica Electrónica. 2010; 32 (3). Disponible en URL: http: //bit.ly/QG98RP

3. Buela CG. Evaluación de la calidad de los artículos y de las revistas científicas: propuesta del factor de impacto ponderado y de un índice de calidad. Psicothema. 2008; 1 (15): 30.

4. Camps D. El análisis bibliométrico de Universitas Scientarium. Universitas Scientiarum. 2009; 14 (1):

5. Ortiz MI y cols. Factor de impacto en las revistas de enfermería. Enfermería Clínica. 2002; 12 (6): 266-267. 
6. Armero B. Análisis cienciométrico de la revista Enfermería Global 2002-2004. Enfermería Global [sine anno]; 7: 2.

7. Gálvez TA y cols. Consumo de información de las revistas de enfermería del área lingüística del español y del portugués en 2002. Desarrollo Científico De Enfermería. 2004; 12 (3): 70.

8. Tomás CV y cols. Estudio bibliométrico de la producción científica y uso de la Revista Chilena de Nutrición a través de la red Scielo (de 2002 a 2007). Revista Chilena de Nutrición. 2010; 37 (3): 331.

9. Serrano GP y cols. Análisis de la producción científica. Revista Metas de Enfermería. 2004; 14 (48-49): 79.
10. Bordons M, Zulueta MA. La actividad científica a través de indicadores bibliométricos. Rev Ese Cardiol. 1999; 52: 790-800.

11. Rodríguez M, Toledo C. Análisis de la producción científica de la revista enfermería, revista Enfermería Neurológica en los años 2007-2009. ENE. 2009; 3 (2): 18.

12. Camps D. Estudio bibliométrico general de colaboración y consumo de la información en artículos originales de la revista Universitas Médica, período 2002 a 2006. Universitas Medica. 2007; 48 (4): 362.

13. Op. Cit. Rodríguez M, Toledo C. :2009:3(2) 17.

14. Figueredo E. Valoración curricular de las publicaciones científicas. Medicina Clínica de Barcelona. 2005; 125 (17): 661-663. 